\title{
Theory of Origin of Water in the Solar system and laws on the origin and development of the universe, solar system, planets and their satellites
}

Baku State University, Azerbaijan Republic, Baku. M. S. Ahmedovich

DOI $<$ 10.26821/IJSHRE.8.3.2020.8302>

$\square$

Abstract-The created cosmogonic theory provides objective solutions to the problems of cosmogony - the origin of the solar

system and the mechanism of the emergence of the Earth's atmosphere and hydrosphere and four giant outer planets during the first stage, the formation of their structure by the reaction of methane and ammonia with oxygen. In the light of modern data from cosmochemistry, geochemistry, and also on the basis of fundamental theoretical research, a previously unknown new phenomenon was discovered that in the Earth's cores and four giant outer planets there is a constant generation of organogenic chemical elements - hydrogen, carbon, nitrogen and oxygen as a result of controlled thermonuclear synthesis from helium (inherited by the predecessor of the solar system - a giant star - and this also proves the origin of the Solar system and the mechanism of planetary origin. The weak intensity of thermonuclear processes is a function of the mass of these planets. This is also evidenced by their heat radiation several times more than they receive it from the sun, as well as their high electromagnetic field and the speed of revolution around its axis and endogenous activity), which explains the mechanism of occurrence of methane, ammonia, carbon dioxide and water in their magma and atmosphere during their existence and at the present time, and which also proves the cause of dehydration planet Earth. The history of the origin of life on Earth, previously erased, has been restored - as a result of the occurrence of water in its primary atmosphere and in an acid-water environment with the ozone layer of the atmosphere and as a result of the chemical and biological evolution of amino acids and proteins (based on sequentially organogenic elements arising in its primary atmosphere hydrogen carbon in the form of carbon dioxide, nitrogen, oxygen, as well as methane and ammonia). It has been revealed that there is a constant cycle of water on the planet Earth, that is, on the one hand, there is its consumption for photosynthetic oxygen, on the other hand, it is generated from hydrogen and oxygen and from the reaction of methane and ammonia with oxygen, as well as in the respiration process of living organisms. This explains the mechanisms of the formation of natural phenomena of cyclones, typhoons, volcanoes and earthquakes during the geological history and at present.

Index Terms-Methane, Ammonia, Oxygen, Magma, Water

\section{I.INTRODUCTION}

This topic has been almost for 300 years, since the publication of the book by E. Kant, "General History and Theory of the Sky," developed jointly by astronomers,

geologists, geochemists, geophysicists, geobiochemists, etc. For example, I will quote from the textbook [2]:

"Modern data on the occurrence of water in the early stages of the Earth's evolution as a planet are based on the latest achievements of our time in the field of astronomy (discoveries and careful study in space of analogues of the solar system, which are at different stages of their development and cosmogonic hypotheses, recently covered in a number of works of scientists [2]). All these authors develop modern aspects of the Emanuel Kant nebular hypothesis which he presented in his work "General History and Theory of the Sky". Consideration of this issue in this section will be carried out on the work of A.A. Marakusheva

"Origin of the Earth and the nature of its endogenous activity" [2].

\section{II.DISCUSSION OF THE PROBLEM OF THE ORIGIN AND OCCURRENCE OF WATER}

Here is a more detailed description of different points of view [2]:

"Simultaneously with the fusion of the "basalt" layer of the earth's crust, the mantle material was degassed. Gaseous components were released from it, which accumulated in the near-Earth space and were held by the force of Earth's gravity. According to the data of the American Scientist G. Yuri, in these early periods of the existence of our planet, its atmosphere was much less dense and had a restorative character. A comparison of it with giant planets (Jupiter, Saturn) suggests that methane, ammonia, to a lesser extent hydrogen, water vapor, carbon dioxide and carbon dioxide predominated in the primary composition of the Earth's atmosphere. Oxygen was practically absent. Condensation of water vapor led to the formation of the first water basins on the Earth's surface. A.P. Vinogradov showed that $1.6 \cdot 10^{24} \mathrm{~g}$ of water was 
released during zone melting of the earth's mantle, that is, almost as much as is contained in modern oceans and seas.

It is believed that already at the end of the lunar stage of development, an ocean could exist, which covered the planet with almost continuous veil. According to another assumption, the ancient hydrosphere of the Earth in its volume was significantly inferior to the modern one. According to G. Yuri, it contained only $10 \%$ of the water volume of existing seas and oceans.

But, in our opinion, the hypotheses of G. Yuri and A. P. Vinogradov, with which it is impossible to agree, do not have a scientific basis and scientific explanation. In recent years, ideas about the possibility of the existence of

"Venusian" conditions on the Earth in the early stages of its geological development have been spreading (V. I. Shuldiner, G. Garrels F. F. Mackenzie, D. Shaw, etc.) [2]. Assume that most of the hydrosphere existing at that time was in a gaseous state and was part of the atmosphere. The latter contained predominantly $\mathrm{H}_{2} \mathrm{O}, \mathrm{CO}_{2}$, and $\mathrm{HCl}$. The atmospheric pressure probably reached $36 \mathrm{MPa}$, and the temperature on the surface of the planet $600^{\circ} \mathrm{C}$. One way or another, but the completion of the lunar stage of the Earth's development was marked by the formation of a "basalt" crust, the emergence of the primary atmosphere and hydrosphere."

However, all the hypotheses of these authors do not indicate the origin and mechanism of the occurrence of water on planet Earth. In addition, in the primary atmosphere of the Earth, they could not predict the existence of its powerful hydrogen shell.

Some authors date the appearance of molecular oxygen in the hydrosphere and atmosphere of the Earth about 2.72.4 billion years ago and determine its photo-sensitized decomposition of water by cyano-bacteria during photosynthesis. But, in our opinion, this oxygen is secondary oxygen.

American geologists [3] write about the discovery of traces of the existence of life on Earth already 4.1 billion years ago, and signs that life could have arisen on our planet not once, but twice, which increases our chances of finding extraterrestrial civilizations and living creatures.

Of interest is the hypothesis of academician A.A. Marakushev that, according to cosmic petrology, the terrestrial planets and the moon are the result of near-solar giant planets that have lost hydrogen shells under the influence of the sun.

However, in our opinion, as an example of the evolution of the planet Earth, this "lost" hydrogen shell is quantitatively consumed for the appearance of water and other chemical compounds in the structure of the Earth, as well as for the evolution of the liquid-hydrogen nickel-iron core of the Earth [7,8]. Because the hydrogen shell of the Earth at the first stage of its formation could not go into outer space due to the high Earth's gravity.

\section{III.DOCTRINE OF COSMOGONY}

\section{Interpretation of the scientific foundations of theory}

Water, therefore, is not only the basis of life on the Earth's biosphere. The ideas about its occurrence on the Earth and in the Solar system are closely connected with the ideas about the origin and evolution of the Earth itself and the Solar system, including the thermodynamics of the corresponding processes. In order to answer these complex questions, it is necessary to clearly present the evolution of the planet Earth itself, that is, its mechanism of stages of occurrence, unlike other planets of the Earth group and four outer giant planets. And for this, we need to look about 4.5 billion years ago in order to find out by what cataclysms and related phenomena the structure of the Earth has evolved. The hypothesis put forward by us says that at this time in the atmosphere of the Earth and four outer giant planets there was high pressure and temperature created by their powerful hydrogen shell (this is a dense layer of hydrogen near the planet space), similar to the chemical composition of the Sun. For this reason, and due to the high gravitational forces, magnetic field and high ultrasonic frequency in their structure (compared to the present time), determined by the formula $\mathrm{F}=\mathrm{m}+\mathrm{k}(\mathrm{F}$ is the gravitational force, $\mathrm{m}$ is the mass of the planet, $\mathrm{k}$ is the superconductivity of the planet - planetary constant), the mass only creates gravity when $\mathrm{k}=0$. When $\mathrm{k}=1, \mathrm{~F}=$ $m+M$, where $M$ is the electromagnetic property of the planet, then mass creates, in addition to gravity, the electromagnetic property of the planet. Four outer giant planets Jupiter, Saturn, Uranus and Neptune, whose electromagnetic properties are even more pronounced, give us reason to believe that thermonuclear processes occur in their nuclei, as in the Earth's core, inherited by the predecessor of the solar system - a giant star - and this also proves the origin and mechanism of the planets of the solar system. The weak intensity of thermonuclear processes is a function of the mass of these planets. This is also evidenced by their heat emission several times greater than they receive it from the Sun, as well as their high speed of revolution around their axis and endogenous activity, which caused an even greater increase in temperature and pressure, in accordance with the laws of thermodynamics and with the emergence of carbon, nitrogen, and oxygen, evolved from helium in the Earth's core and four outer giant planets. Thus, the conditions for combining hydrogen with carbon and nitrogen were created objectively and naturally, as a result of which methane (1) and ammonia (2) arose in their magma and atmosphere:

$$
\begin{aligned}
& \mathrm{C}+2 \mathrm{H}_{2}=\mathrm{CH}_{4}+\mathrm{Q} \\
& \mathrm{N}_{2}+3 \mathrm{H}_{2}=2 \mathrm{NH}_{3}+\mathrm{Q}
\end{aligned}
$$


Subsequent physical conditions, chemical composition, superconducting properties and high reductive oxidative properties of magma and atmosphere (sequentially, that is, first reductive-methane-ammonia, and then many millions of years later water-oxidation eras) led to the emergence of water, by the mechanism of radical branched chain reactions of the resulting methane and ammonia with oxygen in the presence of a lightning discharge during their geological history and at present in their magma and atmosphere according to reactions (3) and (4):

$$
\begin{aligned}
& \mathrm{CH}_{4}+2 \mathrm{O}_{2}=2 \mathrm{H}_{2} \mathrm{O}+\mathrm{CO}_{2}+\mathrm{Q} \\
& 4 \mathrm{NH}_{3}+3 \mathrm{O}_{2}=6 \mathrm{H}_{2} \mathrm{O}+2 \mathrm{~N}_{2}+\mathrm{Q}
\end{aligned}
$$

where the value of $\mathrm{Q}$ indicates the exotherm of these phenomena.

As can be seen from these stoichiometric chemical equations (3) and (4), the amount of Earth's water resources far exceeds its nitrogen resources, which are direct evidence of the occurrence of water on this planet by the indicated mechanism. However, in reality, at present, the calculated value of the nitrogen resources of the Earth's atmosphere far exceeds its water resources. This fact confirms that due to the high thermodynamic stability of molecular nitrogen (dissociation energy of 945 $\mathrm{kJ} / \mathrm{mol}$ ), the intensity of its cycle compared to water is weak and in the subsequent geological era of the accumulation of atmospheric nitrogen resources led to an excess of nitrogen resources. The current 78 percent ratio of nitrogen, the content of which was significantly lower than modern, in the Earth's atmosphere arose/during its geological history as a result of reaction (4). So, the resulting hydrogen, methane, ammonia and oxygen became the cause of the origin of water on the Earth and four outer giant planets. Here it is the philosophy of origin and the mechanism of occurrence of the resources of the atmosphere and hydrosphere of planets in the solar system.

At present, phenomena according to reactions (1) and (2), as well as (3) and (4), also occur in active volcanicseismic processes, as a result of which, according to studies by wellknown volcanologists, during the eruption of volcanoes, the ratio of water to the atmosphere carbon dioxide, which according to equations (3) and (4) corresponds to reality $[4,5]$, and also explains the mechanism of occurrence of organogenic chemical elements as a result of controlled thermonuclear reactions in the Earth's core, in contrast to the Sun's core. Moreover, the phenomena of reaction (1) and (2) occur in magma, and reactions (3) and (4) in magma and the Earth's atmosphere, and this creates tremendous thermal energy and pressure in the magma, explaining the mechanism of occurrence of volcanoes and earthquakes. This also proves the reason for the ongoing endogenous activity of the Earth, during its existence. However, the endogenous activity of the Earth during its primary evolution, until about the Cambrian period, was much higher than modern (compared to the present).

In the recovery-methane-ammonia period of the Earth's evolution, its methane-higher hydrocarbon resources were also formed in its lithosphere on the basis of the carbon and hydrogen produced by reaction (5):

$$
n \mathrm{C}+(2 \mathrm{n}+2) \mathrm{H}_{2}=\mathrm{C}_{\mathrm{n}} \mathrm{H}_{2 \mathrm{n}+2}+\mathrm{Q}
$$

The occurrence of methane and ammonia according to (3) reactions (1) and (2) in the Earth's atmosphere was dictated by the laws of thermodynamics, that is, favorable

(4) physical conditions and chemical composition, which as a result of special physicochemical properties, their temperature and pressure on the Earth were significantly stabilized. It can be said that in the cooling of the surface and the appearance of the Earth's crust of the planet, the main role was played by the reduction-methane-ammonia eras, in which ammonia, as a refrigerant, well cooled the Earth's surface and shielded solar energy until oxygen appeared in the atmosphere.

The fact that a methane-ammonia period of evolution of the Earth's primary atmosphere has arisen is in good agreement and is confirmed by the existence of the atmosphere of four outer giant planets: Jupiter, Saturn, Uranus and Neptune, and some of their satellites: methaneammonia water solutions and their layer of small ice particles [10], proving the common origin and mechanism of the occurrence of water in the solar system. Saturn's rings also consist entirely of the origin of water and a mixture of crystalline water with ammonia, which has always been a mystery to astronomers.

The emergence of the methane-ammonia era also confirms the existence in the Earth's crust of deposits of alkali and alkaline-earth metal nitrates, which arose in the biogeochemical cycle by cyan-bacteria from ammonia, as well as methane-higher hydrocarbon resources.

Also, with the appearance of oxygen in the atmosphere, the ozone layer of the atmosphere was formed in parallel, without which the emergence and evolution of the primitive terrestrial living organisms of the Earth was impossible. At the same time, the emergence of secondary oxygen began in subsequent geological eras, i.e. through photo-sensitized decomposition of water during photosynthesis in the Earth's biosphere, and the content of oxygen and carbon dioxide significantly exceeded their content at present.

On the basis of carbon dioxide, water and light energy, natural conditions were created for the photosynthesis process.

Currently, the reverse phenomenon is occurring in the Earth's atmosphere, when there is an exchange of energy between space and the Earth, during its deceleration and acceleration, occurring mainly in the areas of faults of the 
earth's crust between different tectonic plates [6]. Under the influence of this exchange, at ultrasonic high frequency, electrolysis of water molecules to fuel elements of hydrogen and oxygen and the simultaneous formation of water from the same fuel cells occur. These reactions are accompanied by the formation of hail and lightning and the release of huge thermal energy and pressure, and this two-way process makes itself felt in the form of cyclones, typhoons and other global natural phenomena of the atmosphere, providing the planet with fresh water. In addition, the hydrogen released into the atmosphere (obtained from the Earth's liquidnickel-iron core) [7,8] during the Earth's hydrogen respiration (from the boundary between various tectonic plates), immediately reacts in the presence of a lightning discharge with atmospheric oxygen, forms water.

It has been established that there is a constant circulation of water on the planet, that is, on the one hand, it is consumed in the process of obtaining photosynthetic oxygen, on the other hand, its formation from hydrogen and oxygen fuel cells, as well as in the process of breathing living organisms. Cyclones, especially typhoons, with a capacity of $10^{10}-10^{11} \mathrm{~kW}$ per thousand square kilometers, pour millions of tons of water. The question of where such an amount of water comes from had no answer before our research.

It is curious that, according to Derpolts and Posokhov [4],[5], 1.5 million $\mathrm{km}^{3}$ of free oxygen contained in the atmosphere required decomposition of 1.6 million $\mathrm{km}^{3}$ of water as a result of plant photosynthesis over 3.2 thousand years; and according to Voitikovich's calcûlations [9, 10], over 10 million years, the weight of the Earth's hydrosphere water should be consumed during photosynthesis. And according to the author's calculations, the incessant process of water formation on the planet by reaction (3) and (4) is proved and at present the reason for not dehydrating the planet. Thus, it is possible to calculate the amount of water consumed during photosynthesis during the geological history of the Earth, which is one hundred times the weight of the water of its hydrosphere when the biosphere exists for one billion years. This means that every 10 million years, the weight of the Earth's hydrosphere is updated according to the above mechanism (reactions (3), (4)).

However, at present, in the atmosphere of Venus, there is also a high pressure of about 93 atmospheres and a temperature of about $480^{\circ} \mathrm{C}$, created only by the greenhouse effect of carbon dioxide, which makes up $96.5 \%$ and only $3.5 \%$ nitrogen in its atmosphere [11]. The occurrence of water is theoretically impossible due to the lack of hydrogen in its atmosphere. The lack of endogenous activity in other planets of the Earth group also explains the lack of liquidhydrogen fuel in their core. Due to the absence of the hydrogen envelope of the planet Venus during its primary evolution, the oxygen that was generated was completely consumed in the oxidation of carbon and other elements in the planet's structure, and as a result, its atmosphere was $96.5 \%$ covered with a dense layer of carbon dioxide. In addition, sequential evolution is also evidence of the discovery of the ozone layer in the atmosphere of Venus - the emergence of organogenic chemical elements - hydrogen, carbon, nitrogen and oxygen in the atmosphere of these planets as a result of thermonuclear processes in their nuclei and during their primary evolution.

Hence, the conclusion can be drawn from this that the reason for the appearance of water in the Earth's atmosphere was the existence of its powerful hydrogen shell about 4.5 billion years ago.

The water that appeared in the Earth's atmosphere could not be in a liquid state due to the high exotherm of the above reactions (its occurrence) and the high temperature of the

Earth's surface. It existed only in the form of superheated steam in the Earth's atmosphere. As a result of this process in the atmosphere, water vapor accumulated in huge quantities, and due to a lack of solar energy in the subsequent geological eras, the cooling began on the planet. The first ice age came, which caused cooling and the appearance of a thicker "basalt" layer of the Earth's crust in solid form, which was thinner before the appearance of water. In the reduction-methane-ammonia era, the place of the ozone layer of the Earth's atmosphere was occupied by the ammonia layer, well shielding solar energy. The reason for the hot liquid state of magma was thermonuclear processes in the Earth's core. Probably, the age of the Earth's crust coincides with the age of the occurrence of water in the Earth's atmosphere. These processes completed the first stage of planet Earth formation.

Water arose in the Earth's atmosphere in the form of superheated steam. The subsequent form of its existence is crystalline (cooled by the surface of the planet through vapor-liquid condensation). Over the next era, with an improvement in the absorption of solar energy by the earth's surface, its main liquid form arose - the biological basis of life - water, which exchanges matter and energy with the environment and makes up at least $60 \%$ of the mass of a living cell. The emergence of a liquid form of water also laid the foundation for the emergence of the soil layer of the Earth's crust and the evolution of its biosphere.

So, the reason for the accelerated evolution of life on Earth is the occurrence of the ozone layer in its primary atmosphere and in the water-oxidative environment of organogenic elements - hydrogen, nitrogen, oxygen and carbon in the form of carbon dioxide, methane and ammonia. Favorable thermodynamic physical conditions and chemical composition in the primary atmosphere of the Earth also led to the emergence of both hydrosphere resources and the birth of life. This fact agrees well with the chemical-biological evolution of amino acids and 
proteins on Earth, in contrast to the previously accepted primary chemical composition of the atmosphere according to the well-known theory of the abiogenic origin of life, first argued by A. I. Oparin in 1924 [12]. Only, the indicated author could not take into account that the existence of organic compounds in the absence of its ozone layer is impossible in the primary methaneammonia atmosphere of the Earth's atmosphere. This refutes the hypothesis about the abiogenic origin of life.

The only question remains is how did the polymer chains of proteins and nucleic acids arise, from which the first cells subsequently formed. The main question of the theory of the origin of life is to explain the emergence of ordered informational macromolecule texts from an unordered set of monomers, the emergence of order from chaos. This question is solved by the ability of biopolymers (nucleic acids) to play the role of biosynthetic matrices [13,14].

Water is not only a source of life, but also a raw material for hydrogen energy, which can be verified experimentally by modeling the physical conditions and chemical composition of the Earth's primary atmosphere. The released colossal thermal energy and high pressure during the occurrence of water can be successfully used as an innovative technology for solving the environmental problems of the Earth and Venus atmosphere, as well as for the production of new fuel element compositions for jet and automobile engines, electric power, and heavy-duty humanitarian weapons without damaging this environment and in biospherology for the occurrence of water on the planets Venus and Mars.

\section{IV.CONCLUSION}

According to the created cosmogonic theory, the origin of the biosphere and intelligent life on Earth is the origin of the Earth itself, its location in the solar system, and the fact that the Earth creates a double planetary system with its moon, unlike other planets of the Earth group and four outer giant planets. All of the above reasons, optimal masses and gravitational forces, as well as the remoteness of the Earth from the Sun are the main causes of the occurrence of water and its biosphere. The reason for the absence of the biosphere of the four outer giant planets is the large masses and their remoteness from the Sun, this is evidenced by their high speed of revolution around its axis and endogenous activity. The high speed of revolution around its planet axis is a striking indicator of the high internal energy and electric field arising as a result of thermonuclear processes in their nuclei. Thus, we can say that the "Great phenomenon of the emergence of water in the magma and atmosphere of the Earth" was the first objective fundamental law of the nature of the Earth and will probably behave in a universal manner in exoplanets analogues of the solar system of the Universe. Water will also appear in their magma and atmosphere as a result of the appearance of organogenic chemical elements in thermonuclear processes in their nuclei, that is, while the planet is endogenously active and energy is exchanged between the bowels and the surface, its biosphere may exist. All the above facts in the created theory are described on the basis of exact scientific logic.

V. Laws on the origin and development of the universe, solar system, planets and their satellites

Law 1. The Universe is limitless, constantly expanding periodically in parts by the Big Bangs, arising from their original superhigh dense states, and regaining their former positions of the forms of matter, and according to this cosmogonic teaching - the solar system arose about 14-15 billion years ago - an explosion a giant star, evidence of which is the existence of relic exposure at present in the region of radio waves resulting from the "small explosion" in the solar system.

Law 2. Our little star the Sun is the remainder of this giant star and can be called a secondary star.

Law 3. The explosion of a giant star was preceded by the appearance of planets as debris, fragments of an explosion with various masses that exist even in the gas state. This is evidenced by the existence of outer giant planets in the gas state at present.

Law 4. The resulting planets captured smaller fragments of the explosion outside of Roche, which formed their satellites, which received their constant movement by the magnetosphere of these planets.

Law 5. Mercury and Venus are exceptions to the above rules, which probably existed before the explosion of a giant star. This is evidenced by the absence of their electromagnetic properties, endogenous activity, a very weak revolution around its axis and the absence of their satellites, as well as the absence of methane and ammonia in the atmosphere of Venus.

Law 6. Numerous asteroids, comets and cosmic dust of the solar system are fragments-fragments of the Giant Star.

Law 7. Evidence of this Gigantic Star Explosion is also the existence of external giant planets in the gas state, their high revolution around their axis and electromagnetic field, as well as the current existence of volcanoes on some of their satellites, compared with the inner planets and satellites of the whole solar system.

Law 8. The rotating electromagnetic field of the Sun provides stable rotation of all planets along Copernicus's ellipses in their orbits during their existence according to Kepler's laws based on Newton's law of universal gravitation. Our planet, together with the solar system, does not move along the ellipses of Copernicus, but along 
open trajectories, similar in shape to spirals, at a speed of $250 \mathrm{~km} / \mathrm{s}$

Law 9. The destiny of the entire solar system is determined by two giant planets - Jupiter, Saturn and the Sun. This is evidenced by the mass of Jupiter, which is 318 times greater than the mass of the Earth, and the speed of rotation around the axis 20 times greater than the Earth, and the electromagnetic field on its surface a hundred times greater than on Earth. The mass of Saturn is 95.1 times greater than that of the Earth, and the revolution time around the Sun, equal to 9.5 years, is much less than that of Jupiter, which is 11.9 years. This paradox is associated with the location of Saturn, where the electromagnetic field of the Sun has a maximum volume, which gave a strong acceleration to Saturn compared to Jupiter.

Law 10. A very high speed of revolution around its axis, directed against the rotation of other planets near Uranus, -10 hours 48 minutes - is probably due to the influence of Saturn. On the other hand, Venus has a very slow rotation speed, equal to 369 days, in the opposite direction and the complete absence of electromagnetic properties, endogenous activity and the dense atmosphere of the planet.

Law 11. The planet Mars, whose mass is only 0.108 Earth mass, the speed of revolution around its axis 24 hours 37 minutes, is at a distance of 228 million $\mathrm{km}$ from the Sun. According to the cosmogonictheory[1], the occurrence of water from ammonia and methane occurs in the mantle of Mars: their reaction with the oxygen formed in the core of Mars produces water, carbon dioxide and molecular nitrogen, which is in good agreement with the composition of the real atmosphere of Mars. But only because of the low gravitational force of the planet, these chemical components could not be stored in its atmosphere. This is evidenced by a sufficient electromagnetic property that two satellites can maintain in their orbit, as well as the existence of a high mountain $24 \mathrm{~km}$ high on Mars, indicating high endogenous activity and rotational speed around its axis in its distant geological history.

\section{. REFERENCES}

[1] Aristotle. Composition, in 4 volumes, vol. 3, Moscow: Thought, 1981, 613 p. (Philos. Heritage).

[2] Koronovsky N. V.; Hain V.E; Yasamanov N.A./- Historical Geology: Textbook, 3rd ed., Sr.-M: Publishing Center "Academy", 2008.-464 p.

[3] Bell E.A., Boehnke P., Harrison T. M. and MaoW. L. Potentially biogenic carbon preserved in a 4.1 billion-year-old zircon; National Academy of Sciences 112.47 (2015): 14518-14521, doi: 10.1073 / pnas. 1517557112.

[4] Derpgolts V.F. Water World. - L., Nedra, 1979.-254 p.

[5] Posokhov EV Chemical evolution of the hydrosphere. L.

Gidrometeoizdat. - 1981.-285 p.

[6] Bushuev VV, Kopylov IP, "Space and the Earth, Electromechanical interactions", M, 2005, ed. IAC "Energy", 175 p.
[7] Marakushev A. A., Marakushev S. A., Hydrogen respiration of the Earth - its origin, geological and biological consequences // Alternative Energy and Ecology. 2008. No. 1 (57), 156 - 174 p.,

[8] Marakushev A. A., Marakushev S. A., Origin and fluid evolution of the Earth. Space and Time No. 1 (2010), 98-118 p.

[9] Voitkevich G.V. The origin and chemical evolution of the Earth, M.Nauka, 1973.-168 p.

[10] Voitkevich G.V. Fundamentals of the theory of the origin of the Earth. M.- "Nedra", 1979.-135 p.

[11] Jones B.U., "Life in the Solar System and Beyond," Translation from English. under the editorship of V.G. Surdina, M., Mir, 2007., $336 \mathrm{p}$.

[12] Oparin A.I. Life, its nature, origin and development. -M; 1968.-172 p. [13]Volkonshtein MV, Physics and biology. M., Science, 1980$152 \mathrm{p}$.

[14]Hain V.E., Main problems of modern geology. M., Scientific World, 2003.- $348 \mathrm{p}$

[15] M.S. Ahmedovich., EJERS. European Journal of Engineering Research and Science, Vol. 4, No.10. October 2019 